\title{
Nonlinearity effect on multiwavelength fiber laser based on Sagnac loop mirror interferometer
}

\author{
Abdul Hadi Sulaiman ${ }^{\star 1,2}$, Nur Maizatul Nadia Najamhuri ${ }^{2}$, Muhd Amirul Hafizi Sukor ${ }^{2}$, Aiman \\ Ismail $^{1,2}$, Fairuz Abdullah ${ }^{1,2}$, and Md Zaini Jamaludin ${ }^{1,2}$ \\ ${ }^{1}$ Institute of Power Engineering, Universiti Tenaga Nasional, 43000 Kajang, Selangor, Malaysia \\ ${ }^{2}$ Department of Electrical and Electronics Engineering, College of Engineering, Universiti Tenaga Nasional, 43000 Kajang, Selangor, Malaysia \\ ${ }^{\star}$ Corresponding author: hadisulaiman4@gmail.com
}

Keywords

\author{
multiwavelength fiber laser \\ erbium-doped fiber amplifier \\ Sagnac loop mirror \\ intensity dependent loss
}

Article History

Received: 1 June 2021

Accepted: 8 July 2021

Published: 15 July 2021

\begin{abstract}
This paper demonstrates a multiwavelength fiber laser (MWFL) with erbiumdoped fiber amplifier (EDFA) as gain medium and Sagnac loop mirror interferometer as filter. Stable and flat lasing spectrum was achieved when highly nonlinear fiber (HNLF) was inserted into the laser cavity. The number of lasing lines and extinction ratio (ER) was directly influenced by EDFA gain where at maximum setting, 14 lasing lines with ER of $19 \mathrm{~dB}$ were generated. The stable and flat spectrum achieved spanned about $1.6 \mathrm{~nm}$. This is due to the induced intensity dependent loss mechanism in the cavity by adjusting the half-wave plate of the polarization controller in combination with the HNLF. The MWFL output showed peak power variation of about $2.1 \mathrm{~dB}$ within 100 minutes of observation time.
\end{abstract}

\section{Introduction}

Multiwavelength fiber laser (MWFL) has become one of the main attractions in many scholars, photonics researchers and industries for the application of optical temperature sensor [1], [2]. The generation of multiwavelength spectrum were demonstrated based on several types of gain medium, comb filter and nonlinear effect. Optical amplifiers such as erbium-doped fiber amplifier (EDFA) [3], semiconductor optical amplifier (SOA) [4] and Raman amplifier [5] have been utilized as gain media in multiwavelength laser. For comb filters, researchers used Sagnac loop mirror (SLM) interferometer [6], Fabry-perot filter [7], Mach Zehnder interferometer [8], Lyot filter [9] and array waveguide grating [10] to slice the amplified spontaneous emission (ASE) spectrum to produce the multiwavelength laser.

Although EDFA is an excellent gain medium, its homogeneous gain broadening somewhat limits the number of lasing lines and also caused the peak to be unstable due to high mode competition. One way to reduce this effect is to introduce a nonlinear effect within the cavity so that the mode competition can be suppressed, thus stabilizing the peak of each lasing line. This stabilized peak makes the MWFL lasing lines appear flatter across the spectrum. The common nonlinear effects used are nonlinear polarization rotation (NPR) effect [11], four wave mixing effect [12], stimulated Brillouin scattering [13] and stimulated Raman scattering [14]. In this work, the NPR effect is used to achieve a stable and flat multiwavelength lasing.

Previously, several works on MWFL based on SLM filter were demonstrated utilizing nonlinear device of SOA [4], [15], [16], PC and polarizer [17] and graphene-polymer nanocomposite [18] to achieve a flat and stable multiwavelength spectrum. However, the removal of nonlinear device is not observed as the significant device for the best flatness and stability. In this paper, we demonstrate a MWFL with EDFA as gain medium and SLM interferometer as comb filter. With HNLF removal from the setup, the lasing lines is significantly deteriorated. The best structure produced 14 lasing lines within $5 \mathrm{~dB}$ uniformity and $19 \mathrm{~dB}$ of extinction ratio (ER). The variation of a half-wave plate (HWP) in the SLM interferometer also affect the overall MWFL performance.

\section{Experimental setup}

Figure 1 illustrates the MWFL experimental setup with EDFA as gain medium and SLM interferometer as a comb filter. The EDFA is manufactured from Universiti Putra Malaysia and this module incorporates a microprocessor unit to manage three different operations; automatic gain control, automatic power control (APC) and automatic current control. For amplified spontaneous emission (ASE) operation, only APC mode is allowed as this mode able to 
provide a maximum of $18 \mathrm{dBm}$ power. The laser seed (bottom arrow) is provided by the ASE from the EDFA. A short section of HNLF $(1050 \mathrm{~m})$ increases the nonlinear effect in the cavity. A polarization controller (PC1) was used in combination with $50 \mathrm{~m}$ polarization maintaining fiber (PMF) and 50/50 coupler to create the SLM interferometer. The high birefringence value provided by the PMF creates phase differences in the oscillating signal. A 50/50 splitter divided the light equally to Circulator 1 and Circulator 2. Circulator 1 ensures the light propagates to the EDFA (top arrow) for unidirectional amplification before travelling to Circulator 2 via Port 2 then Port 3. Subsequently, the light travels to the 50/50 coupler to the HNLF. One output port of the 50/50 coupler is connected for observation by an optical spectrum analyzer (OSA), while the other $50 \%$ remains in the cavity for oscillation.

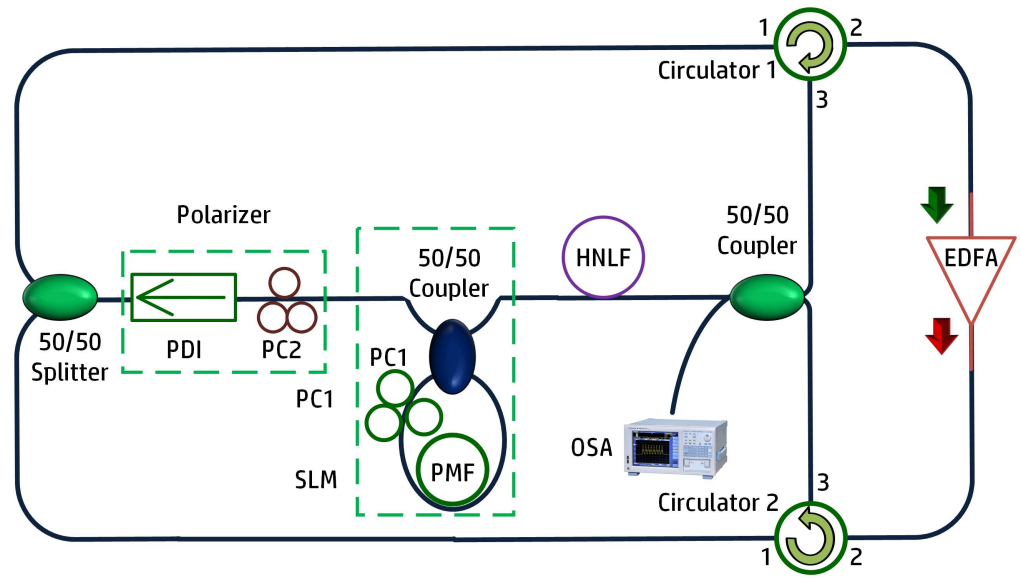

Figure 1. Experimental setup of MWFL based on EDFA and SLM filter

\section{Operating principle}

In this MWFL, the multiwavelength lasing was generated when two counterpropagating lights interacted in the loop containing the PC1 and the PMF. The light propagating in clockwise direction will traverse the PMF before its polarization state was rotated by PC1. The light propagating in a counterclockwise direction had the polarization state rotated before traversing the PMF. This operating caused the light to have different phases and the interaction cause the standing wave to be generated and subsequently the multiwavelength laser upon recombination at the 50/50 coupler [16].

The polarizer and HNLF are used to induce the NPR effect in the cavity. The NPR effect induced can either be intensity-dependent transmission or intensity-dependent loss (IDL) mechanism. The mechanism can be selected by adjusting the polarization controller PC2. To generate stable and flat multiwavelength laser, IDL mechanism is favorable as it functions as an amplitude equalizer whereby the peak of each lasing line will be almost equal, hence the appearance of a flat spectrum.

\section{Results and Discussions}

Figure 2 depicts the ASE signal of the EDFA used. The EDFA is a C-band amplifier ranging from $1510 \mathrm{~nm}$ to $1580 \mathrm{~nm}$ with a typical ASE spectrum with the signature hump around $1530 \mathrm{~nm}$. There is no flattening mechanism in the EDFA as shown in the figure, where the hump around $1530 \mathrm{~nm}$ increases more than the other wavelength as the gain increased.

The MWFL was first observed without HNLF in the cavity. The output spectrum of the laser is depicted in Figure 3 where there is no distinguish lasing peak observed. The peak appears around $1530 \mathrm{~nm}$, overlapping with the hump of the ASE spectrum. This is due to the homogenous gain broadening of the EDFA. When the MWFL with HNLF was observed, it produces 14 flat lasing lines with peak power of $-6 \mathrm{dBm}$ and ER value of $19 \mathrm{~dB}$. This proof that HNLF reduces the mode competition in the cavity from homogenous gain broadening contributed by the EDFA [19]. In previous work of SLM-based MWFL [4], [15]-[18], no comparison was done for with and without HNLF.

The effect of increasing the EDFA output power is shown in Figure 4. The MWFL output spectrum were recorded at three different EDFA powers. At power setting of 10, 14 and $18 \mathrm{dBm}$, the counted lasing lines is 2, 8 and 14 within $5 \mathrm{~dB}$ bandwidth, respectively. It is evident from the figure that the EDFA was able to provide enough gain and the PMF provide the filtering for multiwavelength generation. As the EDFA output power increased, the lasing lines become more stable and flatter. Table 1 summarizes the MWFL performance at each EDFA output power 


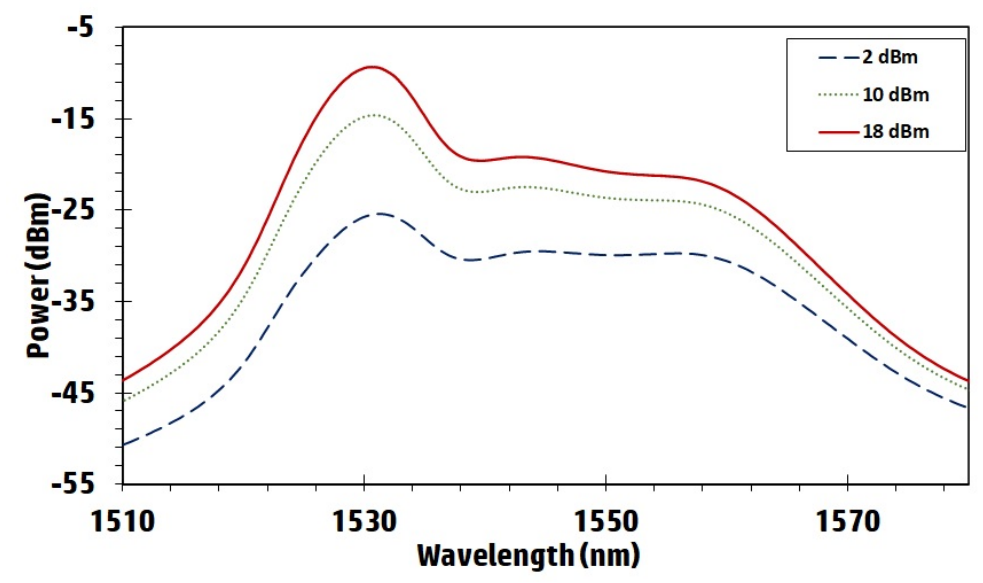

Figure 2. ASE spectrum of the EDFA at different output power settings

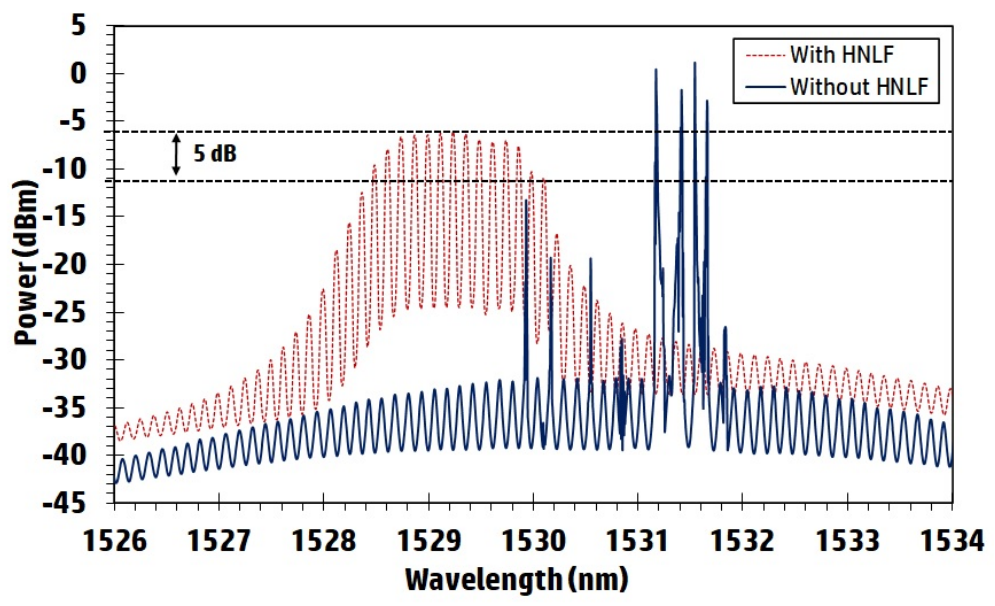

Figure 3. Effect of inserting HNLF into the cavity of MWFL based on EDFA output power setting is $18 \mathrm{dBm}$

setting used. The wavelength range within $5 \mathrm{~dB}$ bandwidth is $0.5,1.0$ and $1.5 \mathrm{~nm}$ according to the increased power setting as in Table 1.

Table 1. The wavelength range, number of lasing lines and ER at different EDFA power

\begin{tabular}{cccc}
\hline EDFA power & $\begin{array}{c}\text { Wavelength range } \\
(\mathbf{n m})\end{array}$ & $\begin{array}{c}\text { Number of lasing } \\
\text { line }\end{array}$ & Extinction ratio (dB) \\
\hline 10 & 0.5 & 2 & 17 \\
14 & 1.0 & 8 & 17 \\
18 & 1.5 & 14 & 19 \\
\hline
\end{tabular}

The angle of HWP in PC1 was varied to study its effect on the lasing output of the MWFL, at the highest EDFA output power setting. The output spectrum at different HWP angle is depicted in Figure 5. When the HWP angle is $0^{\circ}$, there was no lasing line observed. As the angle was increased by $30^{\circ}$ each time, the lasing lines became more stable and flatter. Finally, the most optimum lasing output was observed when the HWP angle is $120^{\circ}$. This different performance at different HWP angle is because of the angle influence the polarization state of the light traveling in the slow and fast axis of the PMF.

A stable laser source is required in an optical sensor system such as a distributed acoustic sensor or similar distributed sensing system since its accuracy can be affected by a slight fluctuation of the source power. Figure 6 depicted the 100-minute stability test of the MFWL laser recorded every 10 minutes. From the observation, the maximum peak power deviation is only $2.1 \mathrm{~dB}$, a value good enough to classify the MWFL as a stable laser source. 

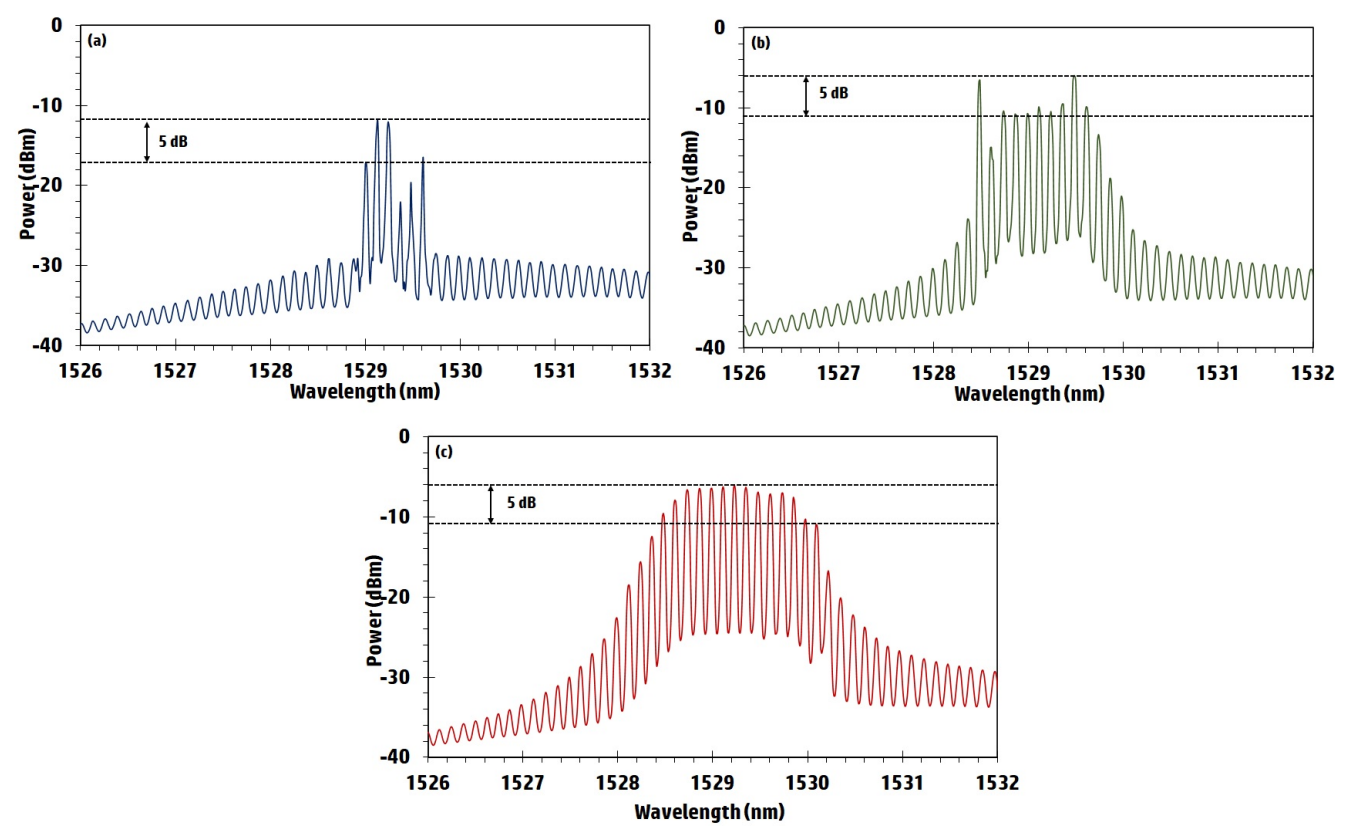

Figure 4. MWFL output spectrum at different EDFA output power setting of (a) $10 \mathrm{dBm}$, (b) $14 \mathrm{dBm}$, and (c) $18 \mathrm{dBm}$

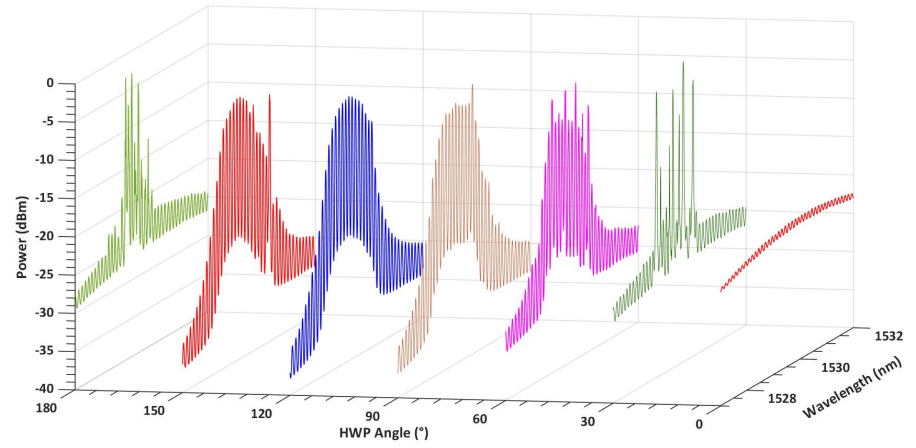

Figure 5. Effect of HWP angle variation on the MWFL output spectrum

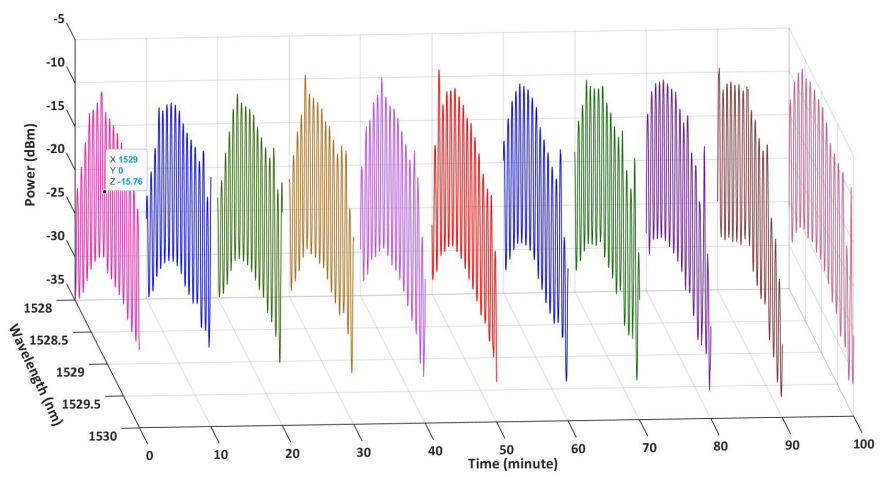

Figure 6. MWFL stability for observation for 100 minutes

\section{Conclusion}

An MWFL with EDFA as gain medium and SLM interferometer as comb filter was successfully demonstrated. The multiwavelength spectrum is flat and stable due to the IDL mechanism as the intensity equalizer, which is induced by the NPR effect obtained from the combination of HNLF and a polarizer. The best performance of multiwavelength spectrum is achieved at EDFA power of $18 \mathrm{dBm}$, generating 14 lasing lines within $5 \mathrm{~dB}$ bandwidth with ER value 
of $19 \mathrm{~dB}$. The HWP position of PC1 is rotated to study its effect on the MWFL performance where $120^{\circ}$ is the optimum angle. The stability test showed that the laser exhibits a mere $2.1 \mathrm{~dB}$ highest peak power fluctuation over 100 minutes of observation.

\section{Acknowledgement}

This research is supported by UNITEN R\& D Sdn. Bhd. via TNB Seed Fund grant number U-TC-RD-19-04.

\section{References}

[1] Z. Xuefang, L. Zengyang, G. Chaoqun, F. Bing, and W. Tianshu, "Multi-wavelength Brillouin erbium-doped fiber laser sensor with high tunable temperature sensing coefficient," Optical and Quantum Electronics, vol. 51, no. 14, pp. 1-14, Dec. 2018, IssN: 1572-817X. Dor: 10.1007/s11082-018-1724-5.

[2] S. Diaz, N. S. Fabian, A. B. Socorro-Leranoz, and I. R. Matias, "Temperature sensor using a multiwavelength erbium-doped fiber ring laser," Journal of Sensors, vol. 2017, pp. 1-6, 2017, IssN: 16877268. DOI: 10.1155/2017/ 8187451.

[3] S. Saleh, N. A. Cholan, A. H. Sulaiman, and M. A. Mahdi, "Stable multiwavelength erbium-doped random fiber laser," IEEE Journal of Selected Topics in Quantum Electronics, vol. 24, no. 3, pp. 1-6, May 2018, IssN: 1077-260X. DoI: $10.1109 /$ jstqe.2017.2759262.

[4] A. H. Sulaiman, N. M. Yusoff, F. Abdullah, and M. A. Mahdi, "Tunable multiwavelength fiber laser based on bidirectional SOA in conjunction with Sagnac loop mirror interferometer," Results in Physics, vol. 18, no. 103301, p. 103 301, Sep. 2020, IssN: 2211-3797. Dor: 10.1016/j.rinp.2020.103301.

[5] Y.-G. Han, C.-S. Kim, J. Kang, U.-C. Paek, and Y. Chung, "Multiwavelength Raman fiber-ring laser based on tunable cascaded long-period fiber gratings," IEEE Photonics Technology Letters, vol. 15, no. 3, pp. 383-385, Mar. 2003, ISSN: 10411135. Dor: 10.1109/lpt.2002.807923.

[6] D.-H. Kim and J. U. Kang, "Sagnac loop interferometer based on polarization maintaining photonic crystal fiber with reduced temperature sensitivity," Optics Express, vol. 12, no. 19, p. 4490, Sep. 2004, IssN: 1094-4087. Dor: 10.1364/opex.12.004490.

[7] C. Yang, L. Xia, Y. Wang, and D. Liu, "Stabilized 51-wavelength erbium-doped fiber ring laser based on high nonlinear fiber," Optics Communications, vol. 318, pp. 171-174, May 2014, IssN: 00304018. DoI: 10.1016/j.optcom.2013.12.077.

[8] F. Wang, X.-L. Zhang, Y. Yu, and X. Huang, "82-channel multi-wavelength comb generation in a SOA fiber ring laser," Optics \& Laser Technology, vol. 42, no. 2, pp. 285-288, Mar. 2010, IssN: 0030-3992. DoI: 10.1016/j.optlastec.2009.07.006.

[9] A. Sulaiman, A. Zamzuri, S. Hitam, A. Abas, and M. Mahdi, "Flatness investigation of multiwavelength SOA fiber laser based on intensity-dependent transmission mechanism," Optics Communications, vol. 291, pp. 264-268, Mar. 2013. Dor: 10.1016/j.optcom.2012.10.078.

[10] H. Ahmad, K. Thambiratnam, A. H. Sulaiman, N. Tamchek, and S. W. Harun, "SOA-based quad-wavelength ring laser," Laser Physics Letters, vol. 5, no. 10, pp. 726-729, Oct. 2008, IssN: 16122011. Dor: 10.1002/lapl. 200810057.

[11] A. H. Sulaiman, N. M. Yusoff, M. Z. A. Kadir, et al., "Investigation on factors influencing flatness of a bidirectional SOA-based multiwavelength fiber laser," Infrared Physics \& Technology, vol. 112, no. November 2020, p. 103 593, Jan. 2021, ISSN: 13504495. DOI: 10.1016/j.infrared.2020.103593.

[12] N. A. Cholan, M. H. Al-Mansoori, A. S. M. Noor, A. Ismail, and M. A. Mahdi, "Multi-wavelength generation by self-seeded four-wave mixing," Optics Express, vol. 21, no. 5, p. 6131, Mar. 2013, IssN: 1094-4087. Dor: 10.1364/oe.21.006131.

[13] H. Ahmad, S. Aidit, and Z. Tiu, "Multi-wavelength Praseodymium fiber laser using stimulated Brillouin scattering," Optics \& Laser Technology, vol. 99, pp. 52-59, Feb. 2018, ISsN: 00303992. DOI: 10.1016/j.optlastec. 2017.09.044.

[14] Z. Wang, T. Wang, W. Ma, Q. Jia, Q. Su, and P. Zhang, "Tunable multiwavelength Brillouin-Raman fiber laser in a linear cavity with spectrum reshaped by Rayleigh scattering," Optical Fiber Technology, vol. 36, pp. 327-333, Jul. 2017, IsSN: 10685200. DoI: 10.1016/j.yofte.2017.04.004. 
[15] M. Zulkifli, N. Hassan, N. Awang, Z. Ghani, S. Harun, and H. Ahmad, "Multi-wavelength fiber laser in the S-band region using a Sagnac loop mirror as a comb generator in an SOA gain medium," Laser Physics Letters, vol. 7, no. 9, pp. 673-676, Jun. 2010, issN: 16122011. Dor: 10.1002/lapl.201010046.

[16] G. Sun, Y. Zhou, L. Cui, and Y. Chung, "Tunable multiwavelength SOA-fiber ring laser based on Sagnac loop mirror incorporating few-mode high birefringence fiber," Laser Physics, vol. 21, no. 11, pp. 1899-1902, Sep. 2011, IssN: 1054-660X. Dor: 10.1134/s1054660x11190261.

[17] Y. Guo, F. Yan, T. Feng, et al., "Switchable multi-wavelength thulium-doped fiber laser using four-mode fiber based Sagnac loop filter," IEEE Photonics Journal, vol. 12, no. 2, pp. 1-10, Apr. 2020, IssN: 19430655. DoI: 10.1109/jphot.2020.2973671.

[18] M. Zhou, Z. Luo, Z. Cai, C. Ye, H. Xu, and J. Wang, "Switchable and tunable multiple-channel erbium-doped fiber laser using graphene-polymer nanocomposite and asymmetric two-stage fiber Sagnac loop filter," Applied Optics, vol. 50, no. 18, pp. 2940-2948, Jun. 2011. Dor: 10.1364/ao.50.002940.

[19] Z. Zhao, X. Li, Y. Li, et al., "Multiwavelength Er-doped fiber laser using an all-fiber Lyot filter," Applied Optics, vol. 57, no. 31, p. 9270, Oct. 2018, issN: 1559-128X. Dor: 10.1364/ao.57.009270. 